Please do not remove this page

RMIT

UNIVERSITY

\title{
Electric tempest in a teacup: the tea leaf analogy to microfluidic blood plasma separation
}

Yeo, Leslie; Friend, James; Arifin, DR

https://researchrepository.rmit.edu.au/esploro/outputs/9921858595401341/filesAndLinks?institution=61RMIT_INST\&index=null

Yeo, L., Friend, J., \& Arifin, D. (2006). Electric tempest in a teacup: the tea leaf analogy to microfluidic blood plasma separation. Applied Physics Letters, 89(10), 1-3. https://doi.org/10.1063/1.2345590

Document Version: Published Version

Published Version: https://doi.org/10.1063/1.2345590

Repository homepage: https://researchrepository.rmit.edu.au

(C) 2006 American Institute of Physics

Downloaded On 2023/04/27 01:33:06 +1000

Please do not remove this page 


\title{
Electric tempest in a teacup: The tea leaf analogy to microfluidic blood plasma separation
}

\author{
Leslie Y. Yeo, ${ }^{\text {a) }}$ James R. Friend, and Dian R. Arifin \\ Micro/Nanophysics Research Laboratory, Department of Mechanical Engineering, Monash University, \\ Clayton, Victoria 3800, Australia
}

(Received 21 May 2006; accepted 12 July 2006; published online 7 September 2006)

\begin{abstract}
In a similar fashion to Einstein's tea leaf paradox, the rotational liquid flow induced by ionic wind above a liquid surface can trap suspended microparticles by a helical motion, spinning them down towards a bottom stagnation point. The motion is similar to Batchelor [Q. J. Mech. Appl. Math. 4, 29 (1951)] flows occurring between stationary and rotating disks and arises due to a combination of the primary azimuthal and secondary bulk meridional recirculation that produces a centrifugal and enhanced inward radial force near the chamber bottom. The technology is thus useful for microfluidic particle trapping/concentration; the authors demonstrate its potential for rapid erythrocyte/blood plasma separation for miniaturized medical diagnostic kits. (C) 2006 American Institute of Physics. [DOI: 10.1063/1.2345590]
\end{abstract}

On describing the role of helical flow in meandering rivers, Einstein drew on the analogy of tea leaves accumulating in the center at the bottom of a stirred tea cup instead of being propelled outwards by the centrifugal force in 1926. ${ }^{1}$ He proposed that this paradoxical behavior arose due to the friction at the base of the cup, thus retarding the rotational velocity and hence the centrifugal force in the small layer of liquid directly above the base (known as the Bödewadt layer ${ }^{2}$ ), as illustrated in Fig. 1. The reduction in the centrifugal force towards the cup bottom therefore resulted in an inward radial velocity and hence recirculation pattern that manifests itself in a bulk helical or swirling flow. In this letter, we report a similar flow phenomenon produced when ionic wind arising from a nearly singular field at the tip of a sharp electrode is employed to drive liquid circulation in a small cylindrical chamber. Colloidal microparticles suspended in the liquid matrix were then convected by the bulk meridional recirculation vortices towards the bottom periphery of the chamber where the swirl-like flow proceeded to drive them towards a stagnation point. This therefore allows the technology to be exploited as a rapid particle trapping or concentration device. In particular, we demonstrate its potential to rapidly separate erythrocytes (red blood cells) from blood plasma, an essential step which approximately takes up to a quarter of the time, cost, and effort in hematological clinical diagnostics and prognostics of diseases and inflammatory responses. This separation procedure is necessary to avoid undesirable matrix effects that lead to nonuniformity and hence inaccurate or irreproducible results. ${ }^{3}$

Efficient separation of bubbles, drops, bacteria, viruses, or blood cells from a sample is unfortunately difficult in microfluidic devices. ${ }^{4,5}$ Strong centrifugation, conventional in laboratory settings, is difficult to achieve at microscale dimensions. The microcentrifugation devices that have been developed are cumbersome, involving the rotation of the entire microfluidic chamber. ${ }^{6,7}$ The use of microfabricated filters, ${ }^{8-10}$ on the other hand, involves complex membranes and complicated fabrication techniques to obtain the requisite small pore sizes. Moreover, large syringe pumps are re-

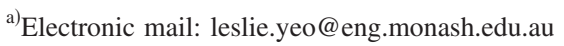

quired to force the fluid through these membranes, negating the advantage of having a microdevice. For blood, the large shear produced by these pumps is counterproductive, resulting in the deformation and denaturing of shear-sensitive erythrocytes. Dielectrophoresis (DEP) has been proposed as a means of particulate concentration and separation to overcome this limitation. ${ }^{11,12}$ Nevertheless, the maximum particle velocities that may be induced via DEP fall quadratically as a function of the particle diameter and is limited below $10 \mu \mathrm{m}$. Moreover, the particle motion is often disrupted by the bulk electrokinetic flow, which can often act in a direction opposite the DEP force. ${ }^{13}$

Application of a potential that exceeds a threshold ionization voltage for air across a sharp (corona) electrode tip mounted $4 \mathrm{~mm}$ above the liquid surface creates a nearly singular electric field in the vicinity of the electrode tip, thereby generating a corona discharge (Fig. 2). ${ }^{14}$ The plasma counterions are propelled away from the electrode, colliding with electroneutral air molecules in the process. An air flow directed towards the liquid surface is thus produced due to the transfer of momentum from these collisions, as depicted in Fig. 2(a). The liquid flow that arises due to interfacial shear depends on the position and angle of inclination of the corona electrode. If the electrode is mounted vertically and is symmetrically centered above the liquid surface, as shown in Fig. 2(b) with $\phi=90^{\circ}$, then air flows outward radially and hence an axisymmetric distribution of radial shear arises at the air-fluid interface. As a consequence, the fluid moves outward radially along the surface, and due to flow conser-

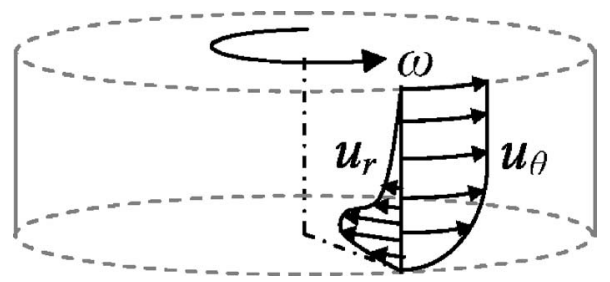

FIG. 1. Azimuthal and radial velocity profiles showing how a recirculating flow arises due to the decrease in the azimuthal velocity and hence centrifugal force and the increase in the inward radial velocity at the fluid layer near the bottom of the chamber. 


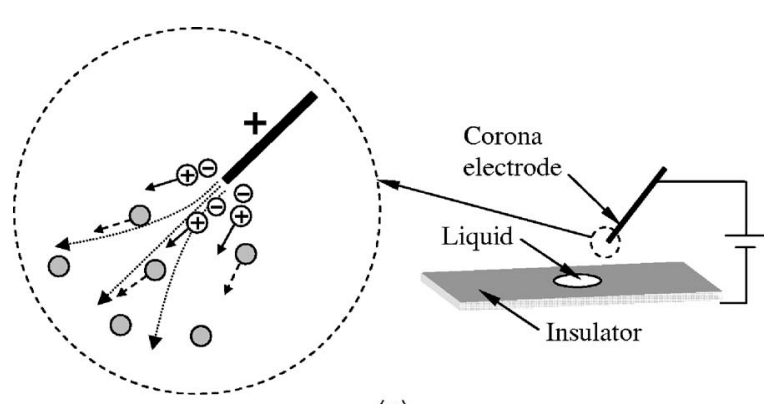

(a)

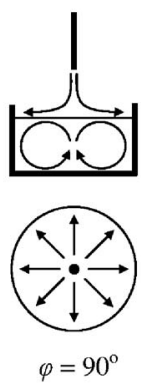

(b)
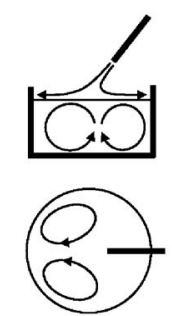

$0<\varphi<90^{\circ}$

(c)

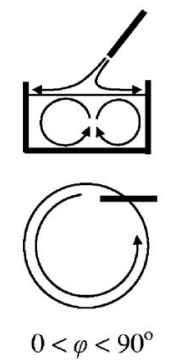

(d)
FIG. 2. (a) Top diagram in panel (a) shows a schematic representation of the experimental setup; the inset shows the mechanism by which ionic wind is generated. The bottom panels show side and plan views of the different surface and bulk liquid flow configurations in which the corona electrode is mounted in a (b) symmetrically centered vertical position $\left(\phi=90^{\circ}\right)$, (c) symmetrically centered inclined position $\left(0^{\circ}<\phi<90^{\circ}\right)$, and (d) laterally inclined position $\left(0<\phi<90^{\circ}\right)$.

vation, it must then recirculate, resulting in internal meridional recirculation vortices in the bulk of the liquid. ${ }^{15}$ If the velocity of the air thrust onto the liquid surface is sufficient, a small interfacial depression of the fluid surface at the center accompanies the secondary recirculating flow. ${ }^{16}$ If the corona electrode is inclined $\left(0^{\circ}<\phi<90^{\circ}\right)$ symmetrically along the centerline as illustrated in Fig. 2(c), however, a different flow behavior arises in which a pair of surface vortices accompanies the meridional recirculation. This surface vortex phenomenon was demonstrated as a potential technique for inducing rapid microfluidic mixing and microparticle concentration and separation. ${ }^{17}$ Furthermore, if the corona electrode is inclined and displaced laterally, as depicted in Fig. 2(d), then only a single circulation vortex arises in conjunction with the internal bulk meridional recirculation.

The meridional recirculation is illustrated in Figs. 1 and 3(a), with radial outward flow in the upper part of the chamber and inward flow along the lower side, induced by the circumferential rotation of the free surface, as shown in Fig. 3(b). A central column of rising fluid is fed by descending fluid in the recirculation in Fig. 3(c); a clearer visualization of the spiraling inflow pattern along the bottom of the chamber can be seen in Fig. 3(d). These figures were obtained by solving the hydrodynamic equations of motion for incompressible fluid flow in cylindrical coordinates $(r, \theta, z)$ for a cylindrical water column of radius $a=4 \mathrm{~mm}$ and height $h$ $=3 \mathrm{~mm}$, as shown in Fig. 2(a); the water viscosity and density were taken to be $1.002 \mathrm{cP}$ and $1000 \mathrm{~kg} / \mathrm{m}^{3}$, respectively. The no-slip boundary condition $\mathbf{u}=0$, where $\mathbf{u}$ is the velocity vector, was imposed at the bottom $(z=0$ for all $r \leqslant a)$ and sidewalls $(r=a$ for $0 \leqslant z \leqslant h$ ) of the cylinder, and an angular velocity $u_{\theta}=r \omega$ with $\omega=0.7 \mathrm{rad} / \mathrm{s}$ was applied at the free liquid surface $z=h$. A mesh of 4400 three-dimensional brick elements was used in a finite element analysis solver ANSYS Downloaded 29 Oct 2006 to 130.194.13.104. Redistribution subject (a)

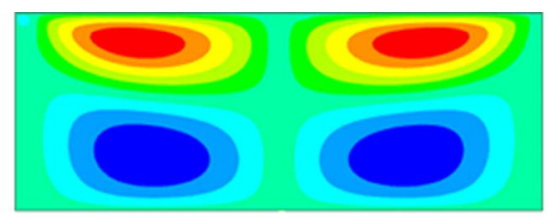

(b)

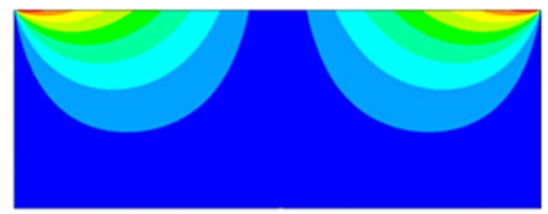

(c)

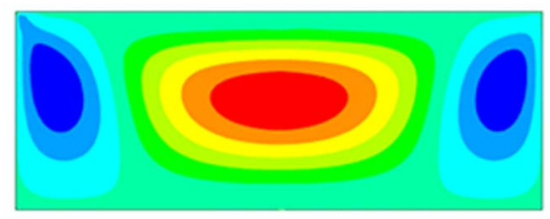

(d)

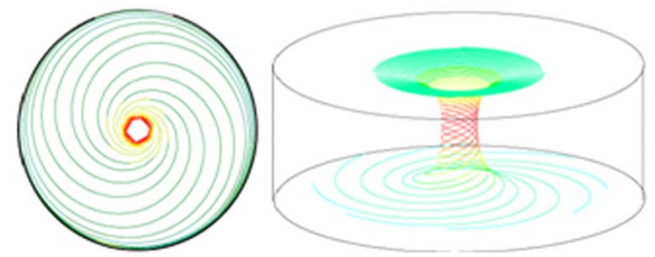

FIG. 3. (Color online) Fluid velocity distribution along a diametral cross section, in the (a) radial $r$, (b) circumferential $\theta$, and (c) vertical $z$ directions; warm colors (red, orange, and yellow) indicate motion along the respective axis directions, while cool colors (light and dark blues) indicate motion against the axis direction. Flow field traces (d) indicate the formation of an inward spiral along the base of the chamber to a rapidly rising central core by the combined meridional and circumferential fluid motion, which acts to concentrate particles suspended within fluid.

for the numerical computation. The flow behavior was observed to be similar to the annular and spiral flow patterns obtained in the flow between rotating and stationary cylindrical disks (Fig. 1), known as Batchelor flows. ${ }^{18,19}$ The helical swirling motion becomes obvious when a constant height cross-sectional slice of a fluid layer close to the bottom is examined, as depicted in Fig. 3(d).

In the experiments, we employ the configuration shown in Fig. 2(d) in which the microfluidic chamber, $8 \mathrm{~mm}$ in diameter and $4 \mathrm{~mm}$ deep, is filled with sheep's blood. In order to facilitate visualization of the flow phenomenon, we restricted the erythrocyte concentration (hematocrit) to $0.4 \%$ by volume by diluting the sample with phosphate buffer saline ( $\mathrm{pH}$ 7.3); a higher hematocrit level overwhelmed the image with erythrocytes and prevented visualization of the particulate flow. Nevertheless, we have verified that the principle remains workable for whole blood ( $40 \%$ hematocrit for adult humans). Figure 4 is a time-sequence image mosaic showing the concentration of erythrocytes at a stagnation point at the bottom of the microfluidic chamber. We intentionally chose a specific parameter set in which the separation took place more slowly for clearer visualization. By optimizing the applied voltage and frequency $(V \sim 0.94 \mathrm{kV}$ rms, $f \sim 90 \mathrm{kHz}$ ), the separation process can, however, be performed relatively quickly in just under $200 \mathrm{~s}$. The separation is efficient, leaving the liquid above to consist of erythrocyte-lean plasma with a hematocrit below $0.003 \%$, deduced by measuring the hemoglobin concentration via UVvis spectroscopy $(150 \mathrm{mg}$ hemoglobin/ml for one erythrocyte). The secondary bulk meridional recirculating flow [top to AlP license or copyright, see http://apl.aip.org/apl/copyright.jsp 


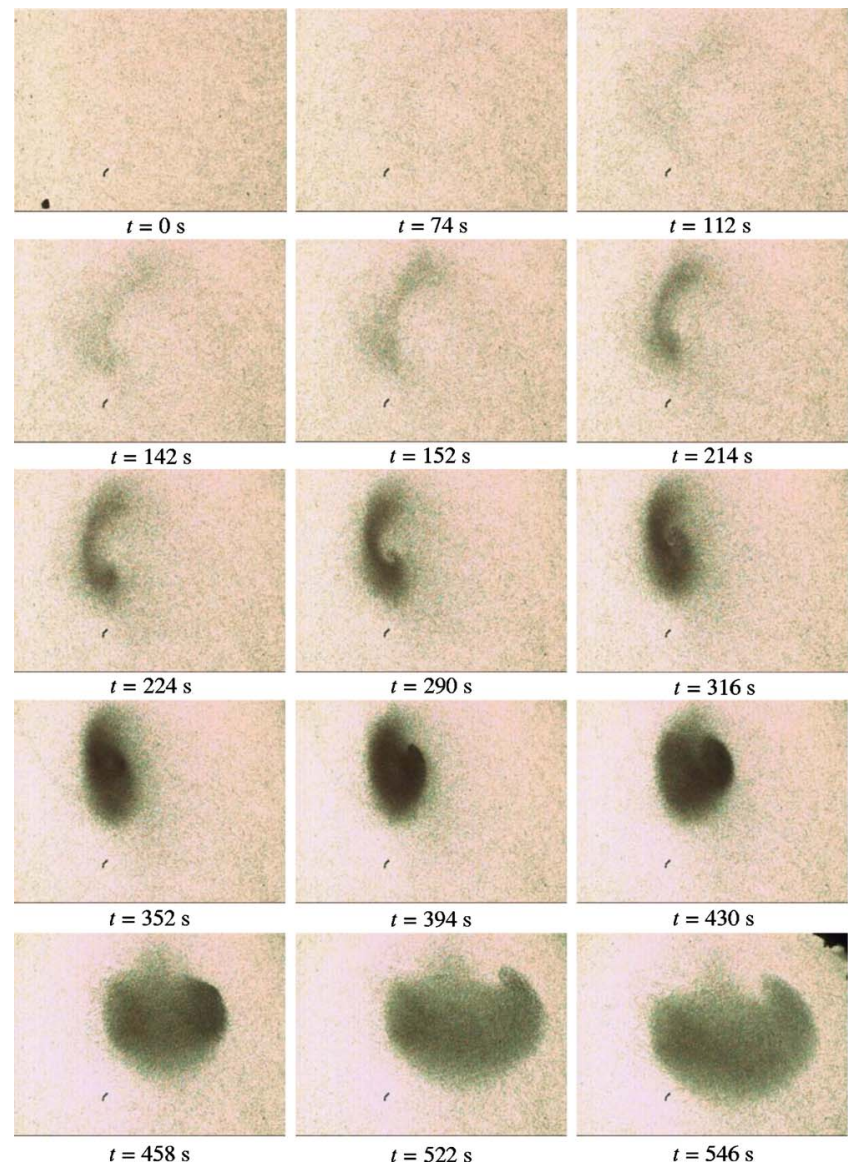

FIG. 4. (Color online) Sequences of images acquired at 60 frames/s showing the trapping of erythrocytes at the bottom of the microfluidic chamber due to the helical flow. The applied voltage and frequency for this particular experiment were $0.56 \mathrm{kV} \mathrm{rms}$ and $60 \mathrm{kHz}$, respectively.

of Fig. 2(d)], which arises as a consequence of the primary azimuthal rotation [bottom of Fig. 2(d)], convects the erythrocytes towards the lateral bottom regions, where the helical flow causes the erythrocytes to subsequently follow an anticlockwise spiral-like trajectory towards a central stagnation point [Fig. 3(d)], as seen from the inverted-comma-like shape of the particle ensemble at the bottom of the chamber.

Finally, the similarity of the present flow with Batchelor flows suggests that boundary layer instabilities observed in the latter deserves some brief mention. If the top surface is rotated above a critical angular velocity, instabilities manifesting in the form of stationary axisymmetric and threedimensional structures appear in the Bödewadt and Ekman (fluid layer adjacent to the top rotating surface and bottom stationary surface, respectively) layers. ${ }^{20,21}$ These instabilities are observed in the present experiments and lead to a breakdown of the vortices at high applied voltages and frequencies where the induced rotational velocities are large. ${ }^{17}$ While these instabilities enhance micromixing by generating intense turbulent mixing eddies, ${ }^{17}$ particle trapping within the vortices as a result of the helical rotation is suppressed. Miniaturized platforms for rapid point-of-care medical diagnostics are becoming feasible in replacing conventional clinical laboratory analysis. The small sample volumes employed in microscale assay techniques not only increase patient comfort and compliance by minimizing the amount of blood extracted but also allow the analysis to be carried out precisely at a fraction of the cost and duration by reducing residence times and eliminating complex and lengthy preparation steps. In addition, automated parallelization, portability, and the possibility of on-time repetitive sampling are other advantages of carrying out such procedures at the microscale. ${ }^{4,5,22}$ To this end, the device in this study should prove useful; it contains no mechanically moving parts and does not require electrode-sample contact, and so the usual problems of mechanical failure and sample contamination through electrolytic reactions or nonspecific adsorption of biological compounds are prevented. Joule heating and the risk of electrocution is avoided through the use of high frequency ac fields. Given that the electric field is predominantly in the gas phase and that the plasma electrolyte solution acts as a highly conducting fluid at constant potential, there is little field penetration into the liquid; the surface charge at the polarized interface is represented by the gas phase field which mainly intersects the conducting surface at right angles to give rise to a dominant normal interfacial field. The confinement of the charge to the interface and the absence of field penetration therefore suggest little adverse effects to the biomolecules. In addition, the minimal current and hence low power required allow the miniaturization of the device to dimensions commensurate with portable medical diagnostic kits.

${ }^{1}$ A. Einstein, Naturwiss. 14, 223 (1926).

${ }^{2}$ U. T. Bödewadt, Z. Angew. Math. Mech. 20, 241 (1940).

${ }^{3}$ C. Blattert, R. Jurischka, I. Tahhan, A. Schoth, P. Kerth, and W. Menz, Proceedings of the 26th Annual International Conference of Engineering in Medicine and Biology Society, San Francisco, CA (IEEE, New York, 2004), pp. 2627-2630.

${ }^{4}$ H. A. Stone, A. D. Stroock, and A. Ajdari, Annu. Rev. Fluid Mech. 36, 381 (2004).

${ }^{5}$ T. M. Squires and S. R. Quake, Rev. Mod. Phys. 77, 977 (2005).

${ }^{6}$ J. Guigan, U.S. Patent No. 4,788,154 (1988).

${ }^{7}$ M. J. Pugia, J. A. Profitt, L. S. Schulman, G. Blankenstein, and R.-P. Peters, Patent No. US2004/021449 (2004).

${ }^{8}$ P. Wilding, J. Pfahler, H. H. Bau, J. N. Zemel, and L. J. Kricka, Clin. Chem. 40, 43 (1994).

${ }^{9}$ J. P. Brody, T. D. Osborn, F. K. Forster, and P. Yager, Sens. Actuators, A 54, 704 (1996).

${ }^{10}$ P. K. Yuen, L. J. Kricka, P. Fortina, N. J. Panaro, T. Sakazume, and P. Wilding, Genome Res. 11, 405 (2001).

${ }^{11}$ H. A. Pohl, Dielectrophoresis (Cambridge University Press, Cambridge, England, 1978).

${ }^{12}$ P. R. C. Gascoyne and J. Vykoukal, Electrophoresis 23, 1973 (2002).

${ }^{13}$ A. R. Minerick, R. Zhou, P. Takhistov, and H.-C. Chang, Electrophoresis 24, 3703 (2003)

${ }^{14}$ L. B. Loeb, Electrical Corona (University of California Press, Berkeley, 1965).

${ }^{15}$ R.-I. Ohyama, K. Kaneko, and J.-S. Chang, IEEE Trans. Dielectr. Electr. Insul. 10, 57 (2003).

${ }^{16} \mathrm{H}$. Kawamoto and S. Umezu, J. Phys. D 38, 887 (2005).

${ }^{17}$ L. Y. Yeo, D. Hou, S. Maheshswari, and H.-C. Chang, Appl. Phys. Lett. 88, 233512 (2006).

${ }^{18}$ G. K. Batchelor, Q. J. Mech. Appl. Math. 4, 29 (1951).

${ }^{19}$ H.-P. Pau, Phys. Fluids 15, 4 (1972).

${ }^{20}$ L. Schouveiler, P. Le Gal, M. P. Chauve, and Y. Takeda, Experientia 26, 179 (1999).

${ }^{21}$ E. Serre, E. Crespo del Arco, and P. Bontoux, J. Fluid Mech. 434, 65 (2001).

${ }^{22}$ M. Toner and D. Irimia, Annu. Rev. Biomed. Eng. 7, 77 (2005). 